\title{
The Updated Status and Future Direction of Immunotherapy Targeting B7-HI/PD-I in Osteosarcoma
}

This article was published in the following Dove Press journal: Cancer Management and Research

\author{
Meng-ke Fan ${ }^{1,2}$ \\ Li-li Qi ${ }^{3}$ \\ Qi Zhang ${ }^{2}$ \\ Ling Wang (D) ${ }^{1,2}$
}

'Department of Orthopedic Oncology, The Third Hospital of Hebei Medical University, Shijiazhuang, Hebei, People's Republic of China; ${ }^{2}$ Orthopedic Research Center, The Third Hospital of Hebei Medical University, Shijiazhuang, Hebei, People's Republic of China; ${ }^{3}$ Department of Pathogenic Biology, Hebei Medical University, Shijiazhuang, Hebei, People's Republic of China
Correspondence: Ling Wang Department of Orthopedic Oncology, The Third Hospital of Hebei Medical University, Shijiazhuang, Hebei, People's Republic of China

Email wangling2016uw@।26.com

\section{Qi Zhang}

Orthopedic Research Center, The Third Hospital of Hebei Medical University, Shijiazhuang, Hebei, People's Republic of China

Tel +86031188602590

Email zq_19865@163.com

\begin{abstract}
Although the mortality rate of osteosarcoma (OS) patients has improved, there are still many unsolved problems concerning how to reduce recurrence and metastasis. In the tumor microenvironment, immune escape plays a more important role in tumor progression and development. Many costimulatory molecules of the B7 family have been reported to be involved in regulating immunological interactions between OS cells and immune cells. Among these molecules, B7-H1 and its receptor, programmed death-1 (PD-1), have been the focus of the fields of tumor immunology and have been recently applied in clinical trials of therapies for several solid tumors. These therapies, referred to as B7-H1/PD-1 checkpoint blockade therapies, are designed to block the interaction between the two molecules. Although the mechanism has been reported in some malignancies, the specific impact of B7H1/PD-1 expression on OS has not been well defined. Here, we review the expression, function, and regulatory mechanism of the B7-H1/PD-1 axis in OS and introduce and compare the advantages and disadvantages of B7-H1/PD-1 immunotherapies in OS.
\end{abstract}

Keywords: osteosarcoma, immunotherapy, B7-H1, PD-1

\section{Introduction}

B7 molecules compose a large family of molecules that are expressed on the cell membrane and that participate in the activation or inhibition of immune cells, especially $\mathrm{T}$ cells. ${ }^{1-3}$ As a group of molecules that provide the second signal, costimulatory molecules, which mainly include B7-1/B7-2 and B7-H2, bind receptors on the surface of $\mathrm{T}$ cells to activate downstream signaling, but coinhibitory molecules, which consist of B7-H1/programmed death-1 (PD-1), B7-H3, B7-H5 and B7-H6, inhibit activated $\mathrm{T}$ cells via specific receptors. ${ }^{4-9}$ Great progress in immunotherapy targeting these molecules has improved the outcomes of cancer patients. The checkpoint of the B7-H1/PD-1 axis has been studied relatively thoroughly in cancer and plays an important role in cancer progression. Immunotargeted drugs, such as pembrolizumab, durvalumab and tremelimumab, have been applied to treat a large number of different tumors, such as non-small cell lung cancer, hepatocellular carcinoma and prostate cancer, in the clinic and have prolonged patient survival and improved patient quality of life. ${ }^{10-13}$

Osteosarcoma (OS) and other pediatric solid tumors arise from abnormal differentiation processes of mesenchymal stem cells and account for less than $1 \%$ of all tumors. ${ }^{14,15}$ These tumors often occur in children and young adults. ${ }^{16,17}$ The treatment 
for OS and other pediatric solid tumors has been greatly improved since the development of neoadjuvant chemotherapy. However, OS recurrence and metastasis rates are still high. ${ }^{18,19}$ The immune checkpoint B7-H1/PD-1 is critical in OS and other pediatric solid tumors. ${ }^{20-22}$ Recent studies have shown that B7-H1/PD-1 monotherapy is less effective and can easily cause many adverse reactions in OS patients. ${ }^{21-28}$ However, combination therapy has a more positive effect on OS in vitro and in vivo. ${ }^{29-31}$ In this review, we will focus on combination therapy strategies for OS and pay close attention to the structure, function, regulation and prognostic value of the B7-H1/PD-1 axis.

\section{Structure, Expression and Functions of B7-HI/PD- I}

B7-H1, known as also known as or CD274, was first identified in the GenBank database in 1999. It is a type I transmembrane protein containing an IgV-like domain, an IgC-like domain, a signal sequence, a transmembrane domain, and intracellular domains encoded by the CD274 gene on human chromosome $9 .^{32}$ B7-H1 is mainly expressed on antigen-presenting cells (APCs) and in some kinds of normal tissues. ${ }^{33,34}$ B7-H1 provides the second signal for effector $\mathrm{T}$ cells, whereas major histocompatibility complex (MHC) molecules recognize T-cell receptor (TCR) as the first signal. ${ }^{35}$ PD-1, also named CD279, functions as a receptor of B7-H1 to transduce signals in effector T cells, was first identified in 1992 and is located on human chromosome $2 .^{36} \mathrm{PD}-1$ regulates the function of immune cells, including $\mathrm{T}$ cells, B cells, natural killer (NK) cells, natural killer $\mathrm{T}$ (NKT) cells, macrophages, and dendritic cells (DCs). ${ }^{37-40}$ Structurally, PD-1 consists of a transmembrane domain, a stalk, an Ig superfamily domain, and an intracellular domain containing an immunoreceptor tyrosine-based inhibitory motif (ITIM) and immunoreceptor tyrosine-based switch motif (ITSM). ${ }^{41}$ When the ligand B7-H1 interacts with the receptor PD-1, the intracellular tyrosines in the ITIM and ITSM are activated by binding with $\mathrm{SH} 2$-domain containing tyrosine phosphatase 1 (SHP-1) and SH2-domain containing tyrosine phosphatase 2 (SHP-2), which then transmit inhibitory B7-H1-derived signals to a variety of signaling proteins, such as PI3K/Akt, STAT3 and STAT5, to inhibit immune cells. ${ }^{41,42}$ Therefore, the B7-H1/PD-1 axis plays an important role in immunosuppression in the tumor microenvironment. However, the effect of the B7-
H1/PD-1 axis needs to be clarified through further exploration.

\section{Prognostic Value and Regulation of B7-HI/PD-I in OS}

In the OS microenvironment, B7-H1/PD-1 has been widely expressed on some kinds of cell types, including tumor cells and immune cells, such as T cells, macrophages, DCs and NK cells. ${ }^{23,37-39}$ In a study of 107 samples, $25 \%$ were positive for both PD-1 and B7-H1 expression, and high B7-H1 expression was significantly associated with tumorinfiltrating lymphocytes (TILs), DCs, NK cells, and eventfree survival. ${ }^{23}$ In the same work, $43.5 \%$ of 69 samples stained positive for B7-H1. Another study suggested that positive B7-H1 expression was significantly correlated with TILs, tumor location and pathological grade. ${ }^{43}$ Additionally, univariate analysis showed that patient survival was prolonged in the high $\mathrm{B} 7-\mathrm{H} 1^{+}$TIL and $\mathrm{CD} 8^{+}$TIL groups and was shorter in the PD- $1^{+}$TIL group, whereas there was no statistical significance. ${ }^{43}$ Nevertheless, B7-H1 expression was relatively significant in predicting OS progression. ${ }^{44}$ Forty-eight percent of metastatic OS microarrays exhibited positive B7-H1 expression in the study by Sundara et al, and PD-1 could be a predictive marker for OS metastasis as determined by receiver operating characteristic (ROC) curve analysis. ${ }^{45}$ Therefore, the levels of B7-H1/PD-1 in OS are quite significant for predicting OS progression and patient prognosis. It is important to further explore the mechanism by which B7-H1/PD-1 promotes OS development.

The B7-H1/PD-1 axis could be regulated by immune cells, stromal cells or their secreted cytokines in the OS microenvironment. Studies have suggested that IFN- $\gamma$ and IL-6 promote B7-H1 expression in vitro. ${ }^{46-48}$ In vivo studies showed that the secretion of IFN- $\gamma$ by $\mathrm{CD}^{+} \mathrm{T}$ cells is predictive of high B7-H1 expression, which further leads to immune tolerance. ${ }^{46}$ Gao et al found that higher PD-1 was expressed on $\mathrm{CXCR}^{+} \mathrm{Tfh}$ cells, which could interact with B7-H1 on OS cells, leading to reduced IL-21 secretion from $\mathrm{CXCR}^{+}$Tfh cells. However, B7-H1 expression could be enhanced indirectly by inducing IFN- $\gamma$ secretion from $\mathrm{CD}^{+} \mathrm{T}$ cells when exogenous IL-21 was added. ${ }^{49}$ B7-H1 expression is also influenced by the interaction of intracellular molecules with various signaling pathways. B7-H1 expression was found to be impaired when histone deacetylase 6 (HDAC6) expression was knocked down or an HDAC6 inhibitor was added. HDAC6 has been shown to 
positively regulate the expression of B7-H1 via the STAT3 pathway. However, B7-H1 upregulation was inhibited if STAT3 was knocked down. ${ }^{48}$ Faciogenital dysplasia 1 (FGD1) is an intracellular protein that binds to phosphatase and tensin homolog deleted on chromosome ten (PTEN) to suppress tumor growth and invasion. In OS, knockdown of FGD1 expression leads to the inhibition of B7-H1 expression. ${ }^{50}$ PTEN downregulation or mutation affects signaling between FGD1 and B7-H1, whereas high PTEN expression negatively regulates $\mathrm{B} 7-\mathrm{H} 1$ expression by interacting with the PI3K/Akt and NF-kB signaling pathways. ${ }^{50}$ Therefore, B7-H1 expression is regulated not only by secreted proteins but also by molecules located on the cell membrane or cytoplasm, which further lead to the activity or inhibition of downstream pathways.

Endogenous noncoding RNAs are an important class of small molecules that influence B7-H1 expression at the posttranscriptional level. ${ }^{51}$ miRNA-200a can bind PTEN mRNA transcripts to induce $\mathrm{B} 7-\mathrm{H} 1$ upregulation indirectly. ${ }^{51}$
However, miR-140 directly binds B7-H1 mRNA transcripts to decrease the expression of $\mathrm{B} 7-\mathrm{H} 1$, as shown by a luciferase reporter assay in a study by $\mathrm{Ji}$ et al. ${ }^{52}$ More importantly, overexpression of miR-140 increases IL-10, IFN- $\gamma$ and TNF- $\alpha$ expression in OS, and high IFN- $\gamma$ expression stimulates the expression of B7-H1. ${ }^{52}$ Upstream of miRNAs, long noncoding RNAs regulate $\mathrm{B} 7-\mathrm{H} 1$ by interacting with miRNAs. Zhang et al revealed that LINC00657 activates B7-H1 expression by suppressing miR-106a levels. ${ }^{53}$ Consequently, there is a closed feedback loop in OS cells in which B7-H1 levels are regulated via multiple pathways (Figure 1).

\section{Therapeutic Strategies for OS Targeting B7-HI/PD-I}

A large number of studies have fully elucidated that the B7-H1/PD-1 axis has a complicated role in tumor development and progression. ${ }^{54,55}$ Nevertheless, the inhibitory

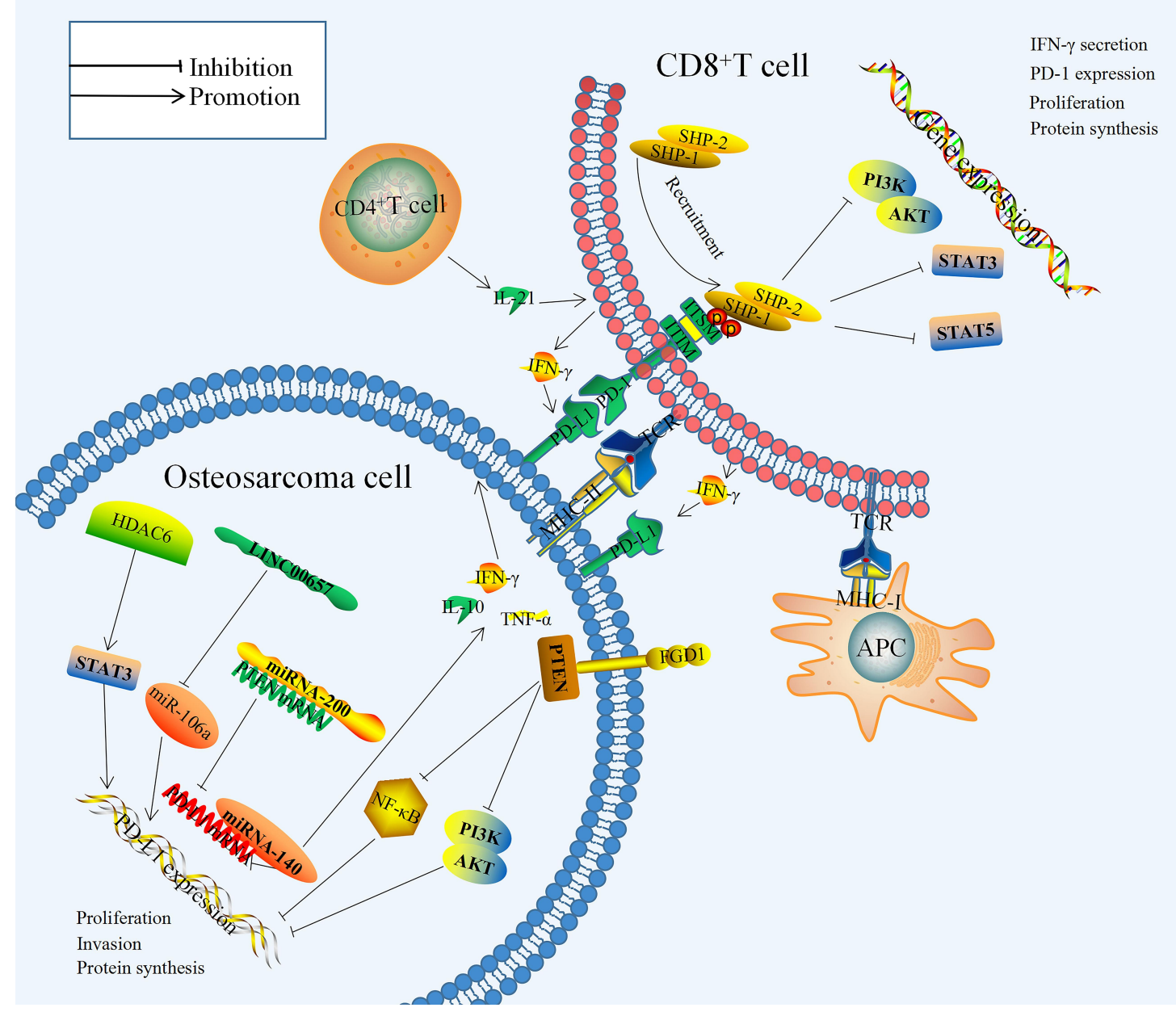

Figure I B7-HI/PD-I signaling pathway in osteosarcoma. 
effects of immune checkpoint inhibitors, including those targeting the B7-H1/PD-1 checkpoint, have been proven to induce positive antitumor responses in diverse cancers. ${ }^{10-13}$ However, the reported effects of these inhibitors on sarcoma are elusive due to the complicated heterogeneity of sarcoma. In studies of OS, anti-B7-H1 /PD-1 treatment alone could have some positive effects, but it could not suppress OS progression or obviously improve patient outcomes. ${ }^{24,46}$ In Davis and his colleagues' study, partial responses (PR) were only observed in patients with Hodgkin lymphoma and non-Hodgkin lymphoma, but were not observed in other tumour types, including OS. ${ }^{56}$ Their clinical observation was similar with those of the Sarcoma Alliance for Research through Collaboration SARC028 study. Only one of the 22 patients with osteosarcoma who were included exhibited PR (5\%). ${ }^{57}$ However, the combination of anti-B7-H1/PD-1 therapy with other adjuvant modalities, such as radiotherapy, chemotherapy or other treatments, has shown appreciable beneficial effects in OS patients. ${ }^{58-60}$ However, related studies and clinical trials are still in the infant stage, and here, we discuss the recent data on the different combinations of B7-H1/PD-1 blockade in OS treatment and the future perspectives of these therapies.

\section{Combination with Chemotherapy}

Various lines of evidence in experimental mouse models and clinical trials support the progress of targeting B7-H1 /PD-1 in improving OS therapy. For example, Wang et al demonstrated that $\mathrm{B} 7-\mathrm{H} 1$ expression was upregulated when doxorubicin was used alone in mice. As expected, the combination of doxorubicin with an anti-B7-H1 antibody could significantly decrease tumor size within the first two weeks and upregulate the proportions of $\mathrm{CD}^{+}$ $\mathrm{T}$ cells, $\mathrm{CD}^{+} \mathrm{T}$ cells and cytotoxic $\mathrm{T}$ lymphocytes (CTLs) in the experimental group. ${ }^{59}$ Markel also showed that 20 $\mu \mathrm{g}$ anti-B7-H1 antibody could decrease Tcell exhaustion to levels observed in the control group, with a corresponding increase in CTLA- 4 expression on cytotoxic T cells in the majority of mice tested. ${ }^{61}$ In another study, Lussier and his colleagues found that the expression of B7-H1 and PD-1 was upregulated in a mouse model of metastatic OS and promoted the immune tolerance of tumor-reactive $\mathrm{T}$ cells toward OS. Although anti-B7-H1 therapy could partially improve $\mathrm{T}$ cell function in vitro and in vivo and prolong survival with fewer pulmonary metastases during disease progression, ultimately, pulmonary disease could unfortunately not be overcome by monotreatment in mice. ${ }^{46}$ This finding also gave us further indication that combinational immunotherapies that block alternative inhibitory receptor pathways on $\mathrm{T}$ cells or other immune cells may lead to more efficient restoration of $\mathrm{T}$ cell function and improve the outcome of metastatic OS.

In addition to mouse OS model results, clinical trial evidence seemed more convincing for the evaluation of the effects of anti-B7-H1/PD-1 therapy combined with chemotherapy in OS patients. Cesne et al launched a Phase 2 clinical trial using pembrolizumab (directly blocking the interaction between B7-H1 and PD-1) in combination with metronomic cyclophosphamide in patients with advanced OS (NCT02406781). These authors showed that four patients had obvious tumor shrinkage and that one patient achieved a partial response (PR). On average, the 6-month nonprogression rate was $13.3 \%$ (95\% confidence interval [CI]: $1.7-40.5) .{ }^{30}$ This result suggested that PD-1 inhibition showed only modest activity in patients with advanced OS. However, we noticed that only 2 of 14 patients with available tumor material showed positive B7$\mathrm{H} 1$ expression, indicating that the nonactive B7-H1/PD-1 pathway correlated with limited benefit from PD-1 blockade in OS. ${ }^{30}$ However, another open-label, phase 2 trial showed positive results concerning a B7-H1 inhibitor combined with apatinib that was applied in OS patients. This trial found that patients with a B7-H1 tumor proportion score $\geq 5 \%$ and pulmonary metastases had a tendency toward a longer PFS than the other patients $(\mathrm{p}=0.004$ and 0.017 , respectively). Patients with positive B7-H1 expression had fewer related toxic effects. ${ }^{31}$ Although immunotherapy for OS did not seem to be as effective as other solid tumors, further clinical trials should explore more joint strategies and implement appropriate correlative studies to better understand the underlying mechanisms.

\section{Combination with Radiotherapy}

Radiotherapy is critical for the treatment of patients with most solid tumors in both curative and palliative settings. Although OS has been considered X-ray-resistant, ${ }^{62}$ a recent innovative technique could deliver modulated high-precision intensity and image-guided radiation therapy; thus, radiation has become an alternative treatment in minimizing the local tumor without excessive damage to surrounding normal tissues. ${ }^{63}$ Therefore, radiation might be a particularly useful therapeutic option for pediatric patients with OS. In a study by Yutaka et al, X-ray irradiation combined with anti-B7-H1 and anti-CTLA-4 antibodies led to faster and more prolonged regression in mouse 
OS tumors than monotherapy, and adding X-ray irradiation significantly increased the probability of the abscopal effect. Moreover, combined therapy has successfully inhibited distant metastasis to some extent and is probably associated with the higher recruitment of CD8 TILs after irradiation and lower Tregs and higher CD8/Tregs. ${ }^{64}$ This result strongly suggested that an optimal radiation delivery regimen can help increase antitumor efficacy. In another model of brain metastatic OS established by Xia and colleagues, there was an apparent improvement in the peripheral blood, including enhanced numbers of $\mathrm{CD}^{+}$ and $\mathrm{CD}^{+} \mathrm{T}$ cells and decreased numbers of myeloidderived suppressor cells (MDSCs) after combination treatment with an anti-PD-1 antibody and radiation therapy. In the microenvironment of OS, T-cell infiltration and IFN- $\gamma$ secretion were promoted concurrently. ${ }^{58}$ Currently, the clinical effect of X-ray irradiation combined with immune checkpoint blockade on OS is still unknown. However, clinical trials with checkpoint blockade therapy have reported beneficial effects in other tumors, including breast cancer, $^{65}$ bladder cancer $^{66}$ and cervical and uterine cancer. ${ }^{67}$ Overall, more clinical data showed that radiation therapy combined with checkpoint inhibition synergizes, which not only enhances antitumor efficacy but also induces the abscopal effect outside of the radiation field.

\section{Combination with Other Targeted Drugs}

It is generally assumed that immune cell-based therapy has the potential to eliminate tumors due to its specialized antigen recognition activity to specifically kill tumors. ${ }^{68}$ However, the effects of this therapy on OS were not satisfactory because of the absence of specific tumor antigens in OS. ${ }^{69}$ Therefore, immune checkpoint inhibitors combined with cell-based immunotherapies would be alternative promising treatment modalities for OS patients. A recent study found that the stromal cell-derived factor $1 / \mathrm{C}-\mathrm{X}-\mathrm{C}$ chemokine receptor type 4 (SDF-1/CXCR4) axis was a crucial factor in recruiting MDSCs into the OS microenvironment and that MDSCs contributed to facilitating immunosuppression and impeding tumor progression by inhibiting a number of activated CTLs in the OS microenvironment. However, positive and effective improvements to the inhibition of tumor growth and prolongation of survival status in mice were observed in the group treated with anti-PD-1 antibody combined with SDF-1/ CXCR4 inhibitor; these outcomes were far better than those of the monotherapy group. ${ }^{60}$ Additionally, Shi et al found that the survival of mice was increased from 37 days to 50 days and that tumor weight decreased by $60 \%$ when tumor-bearing mice were treated with PI3K protein inhibitor and an anti-PD -1 antibody compared with control monotreatment. ${ }^{70}$ L-arginine is an important amino acid for the maintenance of normal immune cellular functions, but it is usually scarce in the tumor environment, leading to T-cell dysfunction and apoptosis. Therefore, He et al showed that L-arginine supplementation showed a trend in strengthening PD-1 expression on $\mathrm{CD}^{+} \mathrm{T}$ cells. Therefore, the combination of L-arginine with an anti-B7-H1 antibody might become an ideal method to suppress OS and promote immunity. Their experimental results confirmed the previous hypothesis that L-arginine combined with an anti-B7-H1 antibody would not only improve mouse survival but also enhance the number and activity of $\mathrm{CD}^{+} \mathrm{T}$ cells, which also inhibits the growth of MDSCs in a significant manner. ${ }^{71}$ Although advanced progress has been made in different experimental trials, there is an urgent need to establish diversified and personalized alternative regimens for OS treatment.

\section{Combination with Other Checkpoint Inhibitors}

Cytotoxic T lymphocyte-associated antigen-4 (CTLA-4) is another checkpoint inhibitor that participates in the inhibition of activated T cells by binding to its ligands, CD80 and CD86. ${ }^{72}$ In checkpoint inhibitor monotherapy, anti-B7-H1 /PD-1 or anti-CTLA-4 antibodies have been shown to be effective in promoting $\mathrm{T}$ cells and suppressing OS malignancy, respectively. ${ }^{73,74}$ However, more progress has been observed in the combined application of immune checkpoint inhibitors with other therapy modules, and similar effects have also been found when different checkpoint inhibitors were combined. ${ }^{75}$ Lussier and his group found that the expression of CD80/CD86 and CTLA-4 was enhanced with anti-B7-H1 treatment. Direct effects, such as the long-term mouse survival rate being approximately $60 \%$ with combination treatment compared to $0 \%$ survival with anti-B7-H1 therapy alone, were observed. ${ }^{75}$ Therefore, dual $\alpha$-CTLA-4/ $\alpha-B 7-H 1$ treatment was adopted in their metastatic OS mouse model, and the effects appeared to be synergistic and better than the use of $\alpha$-CTLA- 4 mAb alone. This result might be attributed to reactivating TIL activities and thus promoting tumor clearance via blockade of nonoverlapping pathways. Similar results were also found in studies by Shimizu et al, who also investigated different combinations of immune checkpoint inhibitors in a metastatic OS model. The results showed that B7-H1/PD1 axis blockade combined with other checkpoint inhibitors 
led to prolonged survival of more than 16 weeks in the experimental group, while the mice in the control groups survived only 80 days. $^{76,77}$ Further evaluation with hematoxylin and eosin (H\&E) and IHC staining showed that the levels of $\mathrm{CD}^{+}$lymphocytes in the combination therapy group were also higher than those in the control group. ${ }^{76,77}$ The combination of multiple checkpoint inhibitors could be significantly more effective than monotherapy usage at present. However, further studies are needed to determine how these combination therapies affect the human body.

\section{Concluding Remarks}

In recent years, immunotherapy has become a promising treatment for cancer. The interaction between B7-H1 and PD-1, which are immune checkpoint molecules that regulate different signaling pathways, is the basis of immunosurveillance. However, research has shown that therapeutic effects were not obvious with anti-B7-H1/PD-1 monotherapy alone, which could not completely inhibit OS development. The occurrence and progression of OS are complicated and could benefit from the effectiveness of combination therapy. This review describes studies that have meaningfully shown that comprehensive treatment is more effective than anti-B7-H1 /PD-1 monotherapy in animal research and clinical trials. This is a critical first step in the development of OS treatments. It is quite likely that multiple drugs will need to be combined to treat OS, further improving patient survival. Nevertheless, a great amount of research in areas such as combination biomarkers for diagnosis and side effects remains to be explored. In the future, molecular and DNA/ RNA research on the regulatory mechanism of B7-H1/PD-1 should be pursued to build the foundation for OS inhibition and/or the control of side effects.

\section{Disclosure}

The authors report no conflicts of interest for this work.

\section{References}

1. Ni L, Dong C. New B7 family checkpoints in human cancers. Mol Cancer Ther. 2017;16(7):1203-1211. doi:10.1158/1535-7163.MCT-16-0761

2. Capece D, Verzella D, Fischietti M, et al. New B7 family checkpoints in human cancers. molecular cancer therapeutics. J Biomed Biotechnol. 2012;2012(2):926321. doi:10.1155/2012/926321

3. Zou W, Chen L. Inhibitory B7-family molecules in the tumour microenvironment. Nat Rev Immunol. 2008;8(6):467-477. doi:10.1038/ nri2326

4. S P B, M S B, Bai A, et al. Differential requirement for CD70 and CD80/ CD86 in dendritic cell-mediated activation of tumor-tolerized CD8 $\mathrm{t}$ cells. J Immunol. 2012;189(4):1708-1716. doi:10.4049/jimmunol.1201271
5. Chen S, G A C, Pritchard TS, et al. Mechanisms regulating PD-L1 expression on tumor and immune cells. J Immunother Cancer. 2019;7 (1):305. doi:10.1186/s40425-019-0770-2

6. Masugi Y, Nishihara R, Hamada T, et al. Tumor PDCD1LG2 (PD-L2) expression and the lymphocytic reaction to colorectal cancer. Cancer Immunol Res. 2017;5(11):1046. doi:10.1158/23266066.CIR-17-0122

7. Lee YH, Martin-Orozco N, Zheng P, et al. Inhibition of the B7-H3 immune checkpoint limits tumor growth by enhancing cytotoxic lymphocyte function. Cell Res. 2017;27(8):1034-1045. doi:10.1038/ cr.2017.90

8. Chen Q, Wang J, Chen W, et al. B7-H5/CD $28 \mathrm{H}$ is a co-stimulatory pathway and correlates with improved prognosis in pancreatic ductal adenocarcinoma. Cancer Sci. 2019;110(2):530-539. doi:10.1111/ cas. 13914

9. Chen Y, Mo J, Jia X, et al. The B7 family member B7-H6: a new bane of tumor. Pathol Oncol Res. 2018;24(4):717-721. doi:10.1007/ s12253-017-0357-5

10. Garon EB, Rizvi NA, Hui R, et al. Supplementary pembrolizumab for the treatment of non-small-cell lung cancer. $N$ Engl J Med. 2015;372 (21):2018-2028. doi:10.1056/NEJMoa1501824

11. Zhu AX, Finn RS, Edeline J, et al. Pembrolizumab in patients with advanced hepatocellular carcinoma previously treated with sorafenib (KEYNOTE-224): a non-randomised, open-label phase 2 trial. Lancet Oncol. 2018;19(7):940-952. doi:10.1016/S1470-2045(18)30351-6

12. A G D, Ulahannan SV, MakorovaRusher O, et al. Tremelimumab in combination with ablation in patients with advanced hepatocellular carcinoma. $J$ Hepatol. 2017;66(3):545-551. doi:10.1016/j. jhep.2016.10.029

13. Mcneel DG, Smith HA, J C E, et al. Phase I trial of tremelimumab in combination with short-term androgen deprivation in patients with PSA-recurrent prostate cancer. Cancer Immunol Immunother. 2012;61(7):1137-1147. doi:10.1007/s00262-011-1193-1

14. Eid JE, Garcia CB. Reprogramming of mesenchymal stem cells by oncogenes. Semin Cancer Biol. 2015;32:18-31. doi:10.1016/j. semcancer.2014.05.005

15. Lye KL, Nordin N, Vidyadaran S, et al. Mesenchymal stem cells: from stem cells to sarcomas. Cell Biol Int. 2016;40(6):610-618. doi:10.1002/cbin.10603

16. ESMO/European Sarcoma Network Working Group. Bone sarcomas: ESMO clinical practice guidelines for diagnosis, treatment and follow-up. Ann Oncol. 2014;25(suppl 3):113-123. doi:10.1093/ annonc/mdu256

17. Ognjanovic S, Olivier M, Bergemann TL, et al. Sarcomas in TP53 germline mutation carriers: a review of the IARC TP53 database. Cancer. 2012;118(5):1387-1396. doi:10.1002/cncr.26390

18. Jaffe N, O S B, Bielack S. Osteosarcoma lung metastases detection and principles of multimodal therapy. In: Cancer Treatment and Research Pediatric and Adolescent Osteosarcoma. Vol. 152. 2010:165-184.

19. Wang Y, Deng X, Yu C. Synergistic inhibitory effects of capsaicin combined with cisplatin on human osteosarcoma in culture and in xenografts. J Exp Clin Cancer Res. 2018;37(1):251. doi:10.1186/ s13046-018-0922-0

20. Torabi A, Amaya CN, Wians FH Jr, et al. PD-1 and PD-L1 expression in bone and soft tissue sarcomas. Pathology. 2017;49 (5):506-513. doi:10.1016/j.pathol.2017.05.003

21. Machado I, López-Guerrero JA, Scotlandi K, et al. Immunohistochemical analysis and prognostic significance of PD-L1, PD-1, and CD8 \pm tumor-infiltrating lymphocytes in Ewing's sarcoma family of tumors (ESFT). Virchows Arch. 2018;472(5):815-824. doi:10.1007/s00428-018-2316-2

22. Kostine M, Cleven AH, de Miranda NF, et al. Analysis of PD-L1, T-cell infiltrate and HLA expression in chondrosarcoma indicates potential for response to immunotherapy specifically in the dedifferentiated subtype. Mod Pathol. 2016;29(9):1028-1037. doi:10.1038/modpathol.2016.108 
23. Koirala P, Roth ME, Gill J, et al. Immune infiltration and PD-L1 expression in the tumor microenvironment are prognostic in osteosarcoma. Sci Rep. 2016;6(1):30093. doi:10.1038/srep30093

24. Zheng B, Ren T, Huang Y, et al. PD-1 axis expression in musculoskeletal tumors and antitumor effect of nivolumab in osteosarcoma model of humanized mouse. J Hematol Oncol. 2018;11(1):16. doi:10.1186/s13045-018-0560-1

25. Rotz SJ, Leino D, Szabo S, et al. Severe cytokine release syndrome in a patient receiving PD-1-directed therapy. Pediatr Blood Cancer. 2017;64(12):Dec. doi:10.1002/pbc.26642

26. McCaughan GJ, Fulham MJ, Mahar A, et al. Programmed cell death-1 blockade in recurrent disseminated Ewing sarcoma. J Hematol Oncol. 2016;9(1):48. doi:10.1186/s13045-016-0278-x

27. Takenaka W, Takahashi Y, Tamari K, et al. Radiation dose escalation is crucial in anti-CTLA-4 antibody therapy to enhance local and distant antitumor effect in murine osteosarcoma. Cancers (Basel). 2020;12(6):1546. doi:10.3390/cancers12061546

28. Toda Y, Kohashi K, Yamada Y, et al. PD-L1 and IDO1 expression and tumor-infiltrating lymphocytes in osteosarcoma patients: comparative study of primary and metastatic lesions. J Cancer Res Clin Oncol. 2020;146(10):1-14.

29. Liu X, He S, Wu H, et al. Blocking the PD-1/PD-L1 axis enhanced cisplatin chemotherapy in osteosarcoma in vitro and in vivo. Environ Health Prev Med. 2019;24(1). doi:10.1186/s12199-019-0835-3.

30. A L C, Marec-Berard P, J Y B, et al. Programmed cell death 1 (PD-1) targeting in patients with advanced osteosarcomas: results from the PEMBROSARC study. Eur J Cancer. 2019;119:151-157. doi:10.1016/j.ejca.2019.07.018

31. Xie L, Xu J, Sun X, et al. Apatinib plus camrelizumab (anti-PD1 therapy, SHR-1210) for advanced osteosarcoma (APFAO) progressing after chemotherapy: a single-arm, open-label, phase 2 trial. $J$ Immunother Cancer. 2020;8(1):e000798. doi:10.1136/jitc-2020000798

32. Dong H, Zhu G, Tamada K, et al. B7-H1, a third member of the B7 family, co-stimulates T-cell proliferation and interleukin-10 secretion. Nat Med. 1999;5(12):1365-1369. doi:10.1038/70932

33. Ding Q, Lu L, Zhou X, Zhou Y, Chou K-Y. Human PD-L1overexpressing porcine vascular endothelial cells induce functionally suppressive human CD4 \pm CD25hiFoxp3 \pm treg cells. J Leukoc Biol. 2011;90(1):77-86. doi:10.1189/jlb.1210691

34. Krempski J, Karyampudi L, M D B, et al. Tumor-infiltrating programmed death receptor- $1 \pm$ dendritic cells mediate immune suppression in ovarian cancer. J Immunol. 2011;186(12):6905-6913. doi:10.4049/jimmunol.1100274

35. Blank C, Mackensen A. Contribution of the PD-L1/PD-1 pathway to T-cell exhaustion: an update on implications for chronic infections and tumor evasion. Cancer Immunol Immunother. 2007;56 (5):739-745. doi:10.1007/s00262-006-0272-1

36. Ishida Y, Agata Y, Shibahara K, et al. Induced expression of PD-1, a novel member of the immunoglobulin gene superfamily, upon programmed cell death. EMBO J. 1992;11(11):3887-3895. doi:10.1002/j.1460-2075.1992.tb05481.x

37. Saudemont A, Jouy N, Hetuin D, et al. NK cells that are activated by CXCL10 can kill dormant tumor cells that resist CTL-mediated lysis and can express B7-H1 that stimulates T cells. Blood. 2005;105(6):2428.

38. Mazanet MM, Hughes CCW. B7-H1 is expressed by human endothelial cells and suppresses $\mathrm{T}$ cell cytokine synthesis. J Immunol. 2002;169(7):3581-3588. doi:10.4049/jimmunol.169.7.3581

39. Conrad C, Gregorio J, Wang YH, et al. Plasmacytoid dendritic cells promote immunosuppression in ovarian cancer via ICOS costimulation of Foxp3( \pm ) T-regulatory cells. Cancer Res. 2012;72 (20):5240-5249. doi:10.1158/0008-5472.CAN-12-2271

40. Pulko V, Harris KJ, Liu X, et al. B7-H1 expressed by activated CD8 $\mathrm{T}$ cells is essential for their survival. $J$ Immunol. 2011;187 (11):5606-5614. doi:10.4049/jimmunol.1003976
41. Keir ME, Butte MJ, Freeman GJ, Sharpe AH. PD-1 and its ligands in tolerance and immunity. Annu Rev Immunol. 2008;26:677-704. doi:10.1146/annurev.immunol.26.021607.090331

42. Parry RV, Chemnitz JM, Frauwirth KA, et al. CTLA-4 and PD-1 receptors inhibit T-cell activation by distinct mechanisms. Mol Cell Biol. 2005;25(21):9543-9553. doi:10.1128/MCB.25.21.95439553.2005

43. Liu P, Xiao Q, Zhou B, et al. Prognostic significance of programmed death ligand 1 expression and tumor-infiltrating lymphocytes in axial osteosarcoma. World Neurosurg. 2019;129:e240-e254. doi:10.1016/j. wneu.2019.05.121

44. Liao Y, Chen L, Feng Y, et al. Targeting programmed cell death ligand 1 by CRISPR/Cas9 in osteosarcoma cells. Oncotarget. 2017;8 (18):30276-30287. doi:10.18632/oncotarget.16326

45. Sundara YT, Marie Kostine AH, Cleven G, et al. Increased PD-L1 and T-cell infiltration in the presence of HLA class I expression in metastatic high-grade osteosarcoma: a rationale for T-cell-based immunotherapy. Cancer Immunol Immunother. 2017;66(1):119-128.

46. Lussier DM, O’Neill L, Nieves LM. Enhanced T-cell immunity to osteosarcoma through antibody blockade of PD-1/PD-L1 interactions. J Immunother. 2015;38(3):96. doi:10.1097/ CJI.0000000000000065

47. Yoshida K, Okamoto M, Sasaki J, et al. Anti-PD-1 antibody decreases tumour-infiltrating regulatory $\mathrm{T}$ cells. BMC Cancer. 2020;20(1):25. doi:10.1186/s12885-019-6499-y

48. Keremu A, Aimaiti A, Liang Z, et al. Role of the HDAC6/STAT3 pathway in regulating PD-L1 expression in osteosarcoma cell lines. Cancer Chemother Pharmacol. 2018;83(10):255-264. doi:10.1007/ s00280-018-3721-6

49. Gao WW, Zhou JJ, Ji B, et al. Evidence of interleukin 21 reduction in osteosarcoma patients due to PD-1/PD-L1-mediated suppression of follicular helper $\mathrm{T}$ cell functionality. DNA Cell Biol. 2017;36 (9):794-800. doi:10.1089/dna.2017.3669

50. Wu W, Jing D, Meng Z, et al. FGD1 promotes tumor progression and regulates tumor immune response in osteosarcoma via inhibiting PTEN activity. Theranostics. 2020;10(6):2859-2871. doi:10.7150/ thno.41279

51. Liu Z, Wen J, Wu C, et al. MicroRNA-200a induces immunosuppression by promoting PTEN-mediated PD-L1 upregulation in osteosarcoma. Aging (Albany NY). 2020;12(2):1213-1236. doi:10.18632/aging.102679

52. Ji X, Wang E, Tian F. MicroRNA-140 suppresses osteosarcoma tumor growth by enhancing anti-tumor immune response and blocking mTOR signaling. Biochem Biophys Res Commun. 2018;495 (1):1342-1348. doi:10.1016/j.bbrc.2017.11.120

53. Zhang J, Chou X, Zhuang M, et al. LINC00657 activates PD-L1 to promote osteosarcoma metastasis via miR-106a. J Cell Biochem. 2020;121(10):4188-4195. doi:10.1002/jcb.29574

54. Sznol M. Blockade of the B7-H1/PD-1 pathway as a basis for combination anticancer therapy. Cancer J. 2014;20(4):290. doi:10.1097/PPO.0000000000000056

55. Wang J, Yuan R, Song W, et al. PD-1, PD-L1 (B7-H1) and tumor-site immune modulation therapy: the historical perspective. J Hematol Oncol. 2017;10(1):34. doi:10.1186/ s13045-017-0403-5

56. Davis KL, Fox E, Merchant MS, et al. Nivolumab in children and young adults with relapsed or refractory solid tumours or lymphoma (ADVL1412): a multicentre, open-label, single-arm, Phase 1-2 trial. Lancet Oncol. 2020;21(4):541-550. doi:10.1016/S1470-2045(20) 30023-1

57. Tawbi HA, Burgess M, Bolejack V, et al. Pembrolizumab in advanced soft-tissue sarcoma and bone sarcoma (SARC028): a multicentre, two-cohort, single-arm, open-label, phase 2 trial. Lancet Oncol. 2017;18(11):1493-1501. doi:10.1016/S1470-2045(17) 30624-1 
58. Liming X, Hao W, Wenkang Q. Irradiation enhanced the effects of PD-1 blockade in brain metastatic osteosarcoma. J Bone Oncol. 2018;12:61-64. doi:10.1016/j.jbo.2018.05.002

59. Wang J, Hu C, Wang J, et al. Checkpoint blockade in combination with doxorubicin augments tumor cell apoptosis in osteosarcoma. J Immunother. 2019;42(9):1.

60. Jiang $\mathrm{K}$, Li J, Zhang J, et al. SDF-1/CXCR4 axis facilitates myeloid-derived suppressor cells accumulation in osteosarcoma microenvironment and blunts the response to anti-PD-1 therapy. Int Immunopharmacol. 2019;75:105818. doi:10.1016/j.intimp. 2019.105818

61. Markel JE, Noore J, E J E, et al. Using the spleen as an in vivo systemic immune barometer alongside osteosarcoma disease progression and immunotherapy with $\alpha$-PD-L1. Sarcoma. 2018;2018:1-13. doi: $10.1155 / 2018 / 8694397$

62. Zuch D, Giang AH, Shapovalov Y, et al. Targeting radioresistant osteosarcoma cells with parthenolide. J Cell Biochem. 2012;113 (4):1282-1291. doi:10.1002/jcb.24002

63. Park B, Yee C, Lee KM. The effect of radiation on the immune response to cancers. Int J Mol Sci. 2014;15(1):927-943. doi:10.3390/ ijms 15010927

64. Takahashi Y, Yasui T, Tamari K, et al. Radiation enhanced the local and distant anti-tumor efficacy in dual immune checkpoint blockade therapy in osteosarcoma. PLoS One. 2017;12(12):e0189697. doi:10.1371/journal.pone.0189697

65. Hu ZI, Ho AY, McArthur HL. Combined radiation therapy and immune checkpoint blockade therapy for breast cancer. Int J Radiat Oncol Biol Phys. 2017;99(1):153-164. doi:10.1016/j.ijrobp.2017.05.029

66. Tree AC, Jones $\mathrm{K}$, Hafeez $\mathrm{S}$, et al. Dose-limiting urinary toxicity with pembrolizumab combined with weekly hypofractionated radiation therapy in bladder cancer. Int J Radiat Oncol Biol Phys. 2018;101 (5):1168-1171. doi:10.1016/j.ijrobp.2018.04.070

67. Tuyaerts S, Van Nuffel AMT, Naert E, et al. PRIMMO study protocol: a Phase II study combining PD-1 blockade, radiation and immunomodulation to tackle cervical and uterine cancer. BMC Cancer. 2019;19(1):506. doi:10.1186/s12885-019-5676-3

68. Rosenberg SA, Restifo NP. Adoptive cell transfer as personalized immunotherapy for human cancer. Science. 2015;348(6230):62-68. doi:10.1126/science.aaa4967
69. Tsukahara T, Kawaguchi S, Torigoe T, et al. Prognostic significance of HLA class I expression in osteosarcoma defined by anti-pan HLA class I monoclonal antibody, EMR8-5. Cancer Sci. 2006;97 (12):1374-1380. doi:10.1111/j.1349-7006.2006.00317.x

70. Shi X, Li X, Wang $H$, et al. Specific inhibition of $\mathrm{PI} 3 \mathrm{~K} \delta / \gamma$ enhances the efficacy of anti-PD1 against osteosarcoma cancer. J Bone Oncol. 2018;16:100206. doi:10.1016/j.jbo.2018.11.001

71. He X, Lin H, Yuan L, et al. Combination therapy with L-arginine and $\alpha$-PD-L1 antibody boosts immune response against osteosarcoma in immunocompetent mice. Cancer Biol Ther. 2017;18(2):94-100. doi:10.1080/15384047.2016.1276136

72. Chen L, Flies DB. Molecular mechanisms of T cell co-stimulation and co-inhibition. Nat Rev Immunol. 2013;13(4):227-242. doi: $10.1038 /$ nri3405

73. Wang SD, H Y L, B H L, et al. The role of CTLA-4 and PD-1 in anti-tumor immune response and their potential efficacy against osteosarcoma. Int Immunopharmacol. 2016;38:81-89. doi:10.1016/j. intimp.2016.05.016

74. Paoluzzi L, Cacavio A, Ghesani M, et al. Response to anti-PD1 therapy with nivolumab in metastatic sarcomas. Clin Sarcoma Res. 2016;6(1):24. doi:10.1186/s13569-016-0064-0

75. Lussier DM, Johnson JL, Hingorani P, et al. Combination immunotherapy with $\alpha$-CTLA- 4 and $\alpha$-PD-L1 antibody blockade prevents immune escape and leads to complete control of metastatic osteosarcoma. J Immunother Cancer. 2015;3(1):21. doi:10.1186/s40425-015$0067-z$

76. Shimizu T, Fuchimoto Y, Okita H, et al. A curative treatment strategy using tumor debulking surgery combined with immune checkpoint inhibitors for advanced pediatric solid tumors: an in vivo study using a murine model of osteosarcoma. J Pediatr Surg. 2018;53 (12):2460-2464. doi:10.1016/j.jpedsurg.2018.08.023

77. Shimizu T, Fuchimoto Y, Fukuda K, et al. The effect of immune checkpoint inhibitors on lung metastases of osteosarcoma. J Pediatr Surg. 2017;52(12):2047-2050. doi:10.1016/j.jpedsurg.2017.08.030

\section{Publish your work in this journal}

Cancer Management and Research is an international, peer-reviewed open access journal focusing on cancer research and the optimal use of preventative and integrated treatment interventions to achieve improved outcomes, enhanced survival and quality of life for the cancer patient.
The manuscript management system is completely online and includes a very quick and fair peer-review system, which is all easy to use. Visit http://www.dovepress.com/testimonials.php to read real quotes from published authors. 